\title{
Malarial Fever Diagnosed and Treated in a Low Risk Endemic Area
}

\author{
Regmi RD, ${ }^{1}$ Basnet ${ }^{1}{ }^{1}$ Jaishwal C, ${ }^{1}$ Pun $S^{1}$ \\ ${ }^{1}$ Jajarkot Hospital, Jajarkot, Nepal. \\ Corresponding Author: \\ Dr Dharma Raj Regmi \\ Jajarkot Hospital, Jajarkot, Nepal \\ Email: rdharma861@gmail.com
}

\begin{abstract}
A case of a 2-year-old female child who initially presented with fever, later diagnosed and successfully treated as Malaria in Jajarkot Hospital in the District of Jajarkot which is a low malarial prevalence region. Patient had been treated with oral antibiotics in a local out of hospital pharmacy but continued to remain febrile. Patient admitted for evaluation of fever and inability to feed. Malaria was confirmed with ICT based Rapid Diagnostic Test after other foci/infection with high degree of suspicion were ruled out. Patient was treated based on the recent national guideline and eventually discharged after showing significant signs of improvement. This isolated case of malaria in a hilly low risk area like Jajarkot emphasizes the need to remain vigilant and adopt a stringent protocol-based diagnostic for diagnosis and treatment of a febrile patient.
\end{abstract}

\section{INTRODUCTION}

Malaria is a mosquito borne infectious disease affecting humans and animals alike caused by a parasitic protozoan belonging to genus Plasmodium that causes symptoms that typically include fever, tiredness, vomiting and headache. In severe cases it can cause, seizures, coma or death. ${ }^{1}$

Child less than five years of age are one of most vulnerable groups affected by malaria. Severe anemia, hypoglycemia, and cerebral malaria are features of severe malaria more commonly seen in children than in adults.

The disease is most commonly transmitted by an infected female anopheles' mosquito. The mosquito bite introduces the parasite from the mosquito's saliva in to a person's blood, the parasite travel to the liver where they mature and reproduce. Five species of plasmodium can infect and be spread by human. Most death are caused by $\mathrm{P}$. falciparum because P. vivax, P. ovale and P. malariae are generally cause cause a milder form of malaria.

\section{CASE REPORT}

A 2-year-old female child came to the outpatient department with complains of fever and cough with decreased feeding for 14 days. Treatment history included two courses of oral antibiotics from a local pharmacy outside Jajarkot hospital. Patient's mother complained of intermittent fever without a fixed pattern for 14 days with higher temperatures ranging from 100-102 F. Fever was accompanied by cough that was non-productive. Patient had no recent travel history and no close contacts with similar complaints. On examination patient was febrile with $101 \mathrm{~F}$ axillary temperature, pulse of 110 bpm, RR of 32 breaths/min. Chest was clear with bilateral equal air entry. Abdomen was mildly distended with moderate hepatosplenomegaly. Neurological examination showed no abnormal signs. Labs on the day of admission showed TC of 28,000 with absolute eosinophilia, $\mathrm{Hb} \%$ of 8.4, normal S Urea, Creatinine Chest Xray did not suggest any abnormal findings.

Patient was admitted to the inpatient ward as a case of Fever under evaluation with Viral fever, Enteric fever, KalaAzar, Typhus fever as differential diagnosis. Empirical IV antibiotics (Ceftriaxone) was started with conservative hydration with IV fluids, acetaminophen. Close monitoring of the vitals showed continual fever in spite of IV 
antibiotics for 4 days. Repeat labs after 4 days of admission showed consistent leukocytosis of $22,000 / \mathrm{mm}^{3}, 45 \%$ Eosinophils, $34 \%$ Neutrophils. and $\mathrm{Hb} \%$ of 7.7. Non-improvement of fever and blood working after 5 days of inpatient treatment warranted the need to look for possibilities of rarer infections non-endemic to the geographical epidemiology. Rapid diagnostic test (RDT) for Malaria was ordered on the $5^{\text {th }}$ day of hospital stay from which patient was found to be positive for malaria on the RDTs. Oral chloroquine was added to the regimen based on the recommendations of local resistance protocol. Signs of improvement were observed during and after antimalarial therapy and child was discharged after total 10 days of hospital admission.

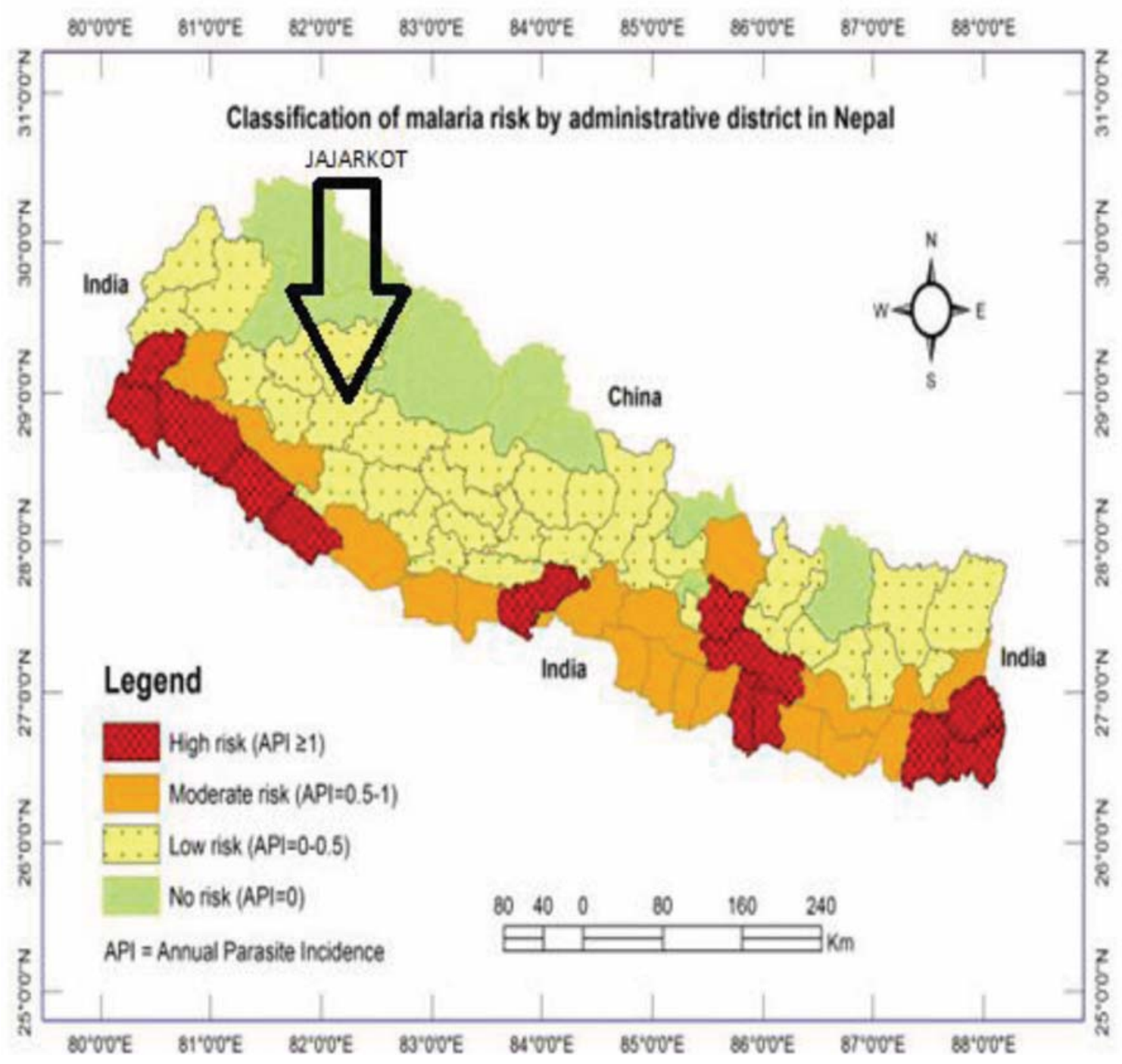




\section{DISCUSSION}

The case of malarial fever is usually not common in non-endemic area. When a case of fever without any recent travel history the diagnosis of malaria is usually missed by the clinician. Most of the time if there is no signs of improvement after few days of admission with empirical treatment of common cause of fever, the case will be referred for fever under evaluation or they can lead in to serious complications (sometimes death). In this case the child's fever and other sign and symptoms were not improved with initial treatment along with the moderate hepatospleenomegaly, anemia and deranged differential blood counts leads us to think about malaria one of the most common cause of high-grade fever.

The WHO estimates that in 2015, 214 million new cases of Malaria resulted in 438,000 deaths worldwide. ${ }^{1}$ The majority of cases $(65 \%)$ occur in children under the 15 years of age. The geographical distribution of Malaria within large region is complex and non-uniform. However, it is prevalent in tropical and subtropical regions due to high yearly precipitation, consistent high temperature, humidity and uncurbed breeding of Anopheles mosquito, the definitive host species that is crucial to reproduction and transmission of the parasite.

In Nepal, about $84 \%$ (23 million) of the people were at risk of malaria in 2012 with at least $4 \%$ at high risk. ${ }^{2}$ One million people lives in areas with a reported incidence of more than one case per 1000 population per year. 64 out of the 75 districts in Nepal are considered to be at risk of malaria infection with 13 districts high risk, 18 moderate risk, 34 low risk, and 10 no risk districts as shown in figure. Jajarkot is a remote mountainous district in Mid-Western region and has been categorized as low risk district for malaria with the last documented case in July 2010.

We, the clinician working in areas where the malarial fever is less likely to occur or in the low risk region for malaria should keep in back of our mind that the malaria could be the cause of fever along with other common cause of it. Early diagnosis and standard treatment protocol will definitely reduce the morbidity and mortality due to malarial fever in any region.

\section{REFERENCES}

1. https://en.wikipedia.org/wiki/Malaria

2. https://www.ncbi.nlm.nih.gov/pmc/articles/PMC4078365/

3. Essential pediatrics by OP Ghai 